Invited commentary

\title{
Primary prevention of diastolic dysfunction in the normal heart: The "Eyes Wide Shut" on a statin pleiotropic effect?
}

\author{
Daniel E. Monopoli*, Maria G. Modena, Giuseppe M. Sangiorgi \\ Department of Cardiology, University Hospital of Modena Via del Pozzo 71 Modena, MO, 41124, Italy
}

\section{A R T I C L E I N F O}

\section{Article history:}

Received 29 January 2011

Accepted 31 January 2011

Available online 12 February 2011

As defined by Braunwald's textbook of Cardiology, diastole includes the period of time during which the myocardium loses its capacity to shorten and returns to an unstressed length [1], accordingly, diastolic dysfunction occurs when this process is prolonged, slowed, or incomplete [2]. Diastolic dysfunction of the left ventricle (DDLV) is thus a pathological condition that may progress to congestive heart failure (HF). Generally, two forms of HF have been identified; i.e., Heart Failure with Normal Ejection Fraction (HFNEF) and Heart Failure with Reduced Ejection Fraction (HFREF) The former, also recognised as Heart Failure with Preserved Ejection Fraction (HFPEF) - a definition which probably better delineates the fact that systolic function is not completely normal in such patients but only apparently preserved [3] is currently observed in about $50 \%$ of HF patients [4,5]. It usually involves the older and the female population, in particular those with a history of hypertension [5-8], and its incidence and prevalence has increased with worsening morbidity and mortality [5,9]. Conversely, patients with HFREF are more likely to be younger, male and to have an ischaemic aetiology compared with HFNEF patients [5]. The European Society of Cardiology (ESC) recently underlined the clinical importance and complexity of HFNEF, and issued new definition criteria, based on clinical signs and/or symptoms of HF, echocardiographic and biological parameters [10].

In this context, diastolic dysfunction refers to mechanical and functional abnormalities present during relaxation and filling of the left ventricle (LV), such as a prolonged isovolumic LV relaxation, slow LV filling, and increased diastolic LV stiffness [11]; whereas HFNEF refers to the clinical syndrome in which patients with HF have little or no ventricular dilatation, preserved EF, and significant, often dominant, diastolic dysfunction. Diastolic dysfunction can thus, be quantified with indices of LV pressure decline and filling. Abnormal pressure decline is characterized by decreased peak

\footnotetext{
* Corresponding author. Tel.: +39 059 4225729; fax: +39 0594222843.

E-mail address: danielmonopoli@libero.it (D.E. Monopoli).
}

- $\mathrm{dP} / \mathrm{dt}$, prolonged isovolumic time constant $(\tau)$, and increased isovolumic relaxation time. Abnormal filling is characterized by slow and incomplete filling, increased atrial contribution to filling, and increased chamber stiffness, which can be caused by abnormalities in both cardiomyocytes and extracellular matrix (ECM) [11-14]. Changes in the amount, composition, and geometry of such ECM proteins, such as collagen and elastin, can alter LV stiffness and causes decreased or delayed relaxation, slow or incomplete filling, and increased diastolic stiffness, leading to the development of HFNEF [12]. Indeed, stiffness of the ECM is largely determined by tissue collagen amount, which in turn depends on the regulatory mechanisms of its synthesis and degradation, relative quantity of collagen type I, and degree of collagen cross-linking [11]. Moreover, cardiac inflammation can also contribute to diastolic dysfunction by triggering the accumulation of ECM through the induction of collagen gene expression and inhibition of cardiac degradation system [15].

Modern evidence-based therapy for HF has shown an improvement in prognosis of patients affected by HF with reduced left ventricular ejection fraction (HFREF). Our group reported the beneficial pharmacological effect of the combination of an aldosterone inhibitor with an angiotensin-converting enzyme inhibitor limiting the synthesis of collagen and reducing the post-infarct remodeling [16]. On the contrary, the prognosis of patients with HFNEF has remained unchanged despite the use of same class pharmacological agents [5,17-21].

The 3-hydroxy-3-methylgutaryl-CoA reductase inhibitors (statins) are well-known potent lipid lowering agents. Additionally to their primary effects, statins have been shown to have several pleiotropic effects on the cardiovascular system [22], including antiinflammatory, antioxidative, and endothelial protective effects. Controversial results have been reported with statin administration in HFNEF. Some experimental and clinical studies have suggested a beneficial effect after statin therapy in HFNEF [23-27]. Fukuta and co-authors were the first to report that in patients with HFPEF statins was associated to improved 
survival [23]. Conversely, another study in animal model showed no effect on the progression of HF neither on survival, despite the attenuation of myocardial fibrosis and improved diastolic stiffness observed in the group treated with statins [28]. Moreover, a recent prospective trial by Gissi-HF-Investigators did not show differences in survival between daily statin therapy in comparison to placebo in patients with chronic HF, including patients with preserved systolic function [29].

In this issue of the Journal, Lerman and associates performed a study in which three groups of pigs ( $n=6$ each) were sacrificed after 12 weeks of normal diet, hypercholesterolemic (HC) diet, and HC diet with simvastatin $(80 \mathrm{mg} /$ day $)$ treatment, respectively. Cardiac function was assessed by electron beam computed tomography and myocardial vascular fraction by micro-computed tomography. Histologic evaluations included collagen content quantification by Sirius Red staining and a hydroxyoproline-based assay. The authors observed that LDL serum concentration was significantly higher in HC diet group, and in HC diet treated with simvastatin compared to normal diet group. Cardiac early diastolic filling was reduced in HC diet group compared with normal diet group and "preserved" in HC diet with simvastatin treatment group. In addition, myocardial vascular fraction and myocardial collagen content was higher in HC group but not in normal diet group and in HC diet with simvastatin group. Immunoblotting showed an increase in myocardial expression of b-FGF, VEGF and TGF- $\beta 1$ in HC diet group compared with normal group while the expression was attenuated in the HC diet with simvastatin treatment group.

How we can we move from bench to bedside after reading the study done by Lerman and co-authors? First, this study provides additional evidences that experimental hypercholesterolemia leads to diastolic dysfunction due to increase myocardial collagen content, especially in the perivascular compartment, along with upregulation of pro-fibrotic and proangiogenetic pathways. Second, as also stated by the authors, the present study shows for the first time in a large size animal model, that administration of a statin prevents these myocardial morphological changes independently of any lipid-lowering effect. These results represent an evidence to support the model of hypercholesterolemia-induced diastolic dysfunction as a reactive process amenable to the nonlipid-lowering properties of statins. Third, the non-lipid-lowering properties of statins already reported in both animal and clinical studies of LV hypertrophy are touhgt to result from mild statin-induced reduction of blood pressure, alterations in myocardial growth regulatory signal transduction pathways, changes in inflammatory or immune-mediated systems, or increased arterial compliance $[30,31]$. Statins may alter arterial compliance by changing the composition of the vascular wall or by changing endothelial response to local and circulating vasoactive compounds and neurohormones [32]. The present study suggests another lipidindependent property, administration of statins could prevent the increase in myocardial collagen content at the bottom of diastolic dysfunction.

Albeit this study opens a new horizon in the field of diastolic dysfunction prevention showing on one side the "indirect" role of hypercholesterolemia in promoting diastolic dysfunction and, on the other side, the ability of sinvastatin for preserving normal diastolic function through a mechanism of action independent of cholesterol reduction, it is hard to believe that lipid lowering therapy will be clinically relevant in normalizing LV structural changes or LV function in patients with HFNEF [32]. Finally, we should keep in mind that these results are based on animal models, and at this moment should be applied with caution to human. Given the important public health impact of diastolic dysfunction, large prospective clinical trials should be performed to evaluate the therapeutic potential of statins in this important field of prevention. - only in this way we could look deeply into this topic with "eyes wide opened".

\section{References}

[1] Opie LH. Mechanisms of cardiac contraction and relaxation. In: Libby P, Bonow RO, Mann DL, Zipes D, editors. Braunwald's Heart Disease. A Textbook of Cardiovascular Medicine. 8th Ed. Philadelphia, PA: Saunders Elsevier; 2008. p. 529-40.

[2] Zile MR, Brutsaert DL. New concepts in diastolic dysfunction and diastolic heart failure, part I: diagnosis, prognosis, and measurements of diastolic function. Circulation 2002;105:1387-93.

[3] Task Force for Diagnosis and Treatment of Acute and Chronic Heart Failure 2008 of European Society of Cardiology, Dickstein K, Cohen-Solal A, Filippatos G, McMurray JJ, Ponikowski P, Poole-Wilson PA, Strömberg A, van Veldhuisen DJ, Atar D, Hoes AW, Keren A, Mebazaa A, Nieminen M, Priori SG, Swedberg K; ESC Committee for Practice Guidelines, Vahanian A, Camm J, De Caterina R, Dean V, Dickstein K, Filippatos G, Funck-Brentano C, Hellemans I, Kristensen SD, McGregor K, Sechtem U, Silber S, Tendera M, Widimsky P, Zamorano JL.ESC Guidelines for the diagnosis and treatment of acute and chronic heart failure 2008: the Task Force for the Diagnosis and Treatment of Acute and Chronic Heart Failure 2008 of the European Society of Cardiology. Developed in collaboration with the Heart Failure Association of the ESC (HFA) and endorsed by the European Society of Intensive Care Medicine (ESICM). Eur Heart J 2008;29(19):2388-442.

[4] Hogg K, Swedberg K, McMurray J. Heart failure with preserved left ventricular systolic function: epidemiology, clinical characteristics, and prognosis. J Am Coll 2004;43:317-27

[5] Owan TE, Hodge DO, Herges RM, Jacobsen SJ, Roger VL, Redfield MM. Trends in prevalence and outcome of heart failure with preserved ejection fraction. $\mathrm{N}$ Engl J Med 2006;355:251-9.

[6] Yancy CW, Lopatin M, Stevenson LW, De Marco T, Fonarow GC. Clinical presentation, management, and in-hospital outcomes of patients admitted with acute decompensated heart failure with preserved systolic function: a report from the Acute Decompensated Heart Failure National Registry (ADHERE) Database. J Am Coll Cardiol 2006;47:76-84.

[7] Fonarow GC, Stough WG, Abraham WT, et al. Characteristics, treatments, and outcomes of patients with preserved systolic function hospitalized for heart failure: a report from the OPTIMIZE-HF Registry. J Am Coll Cardiol 2007;50:768-77.

[8] Kitzman DW, Gardin JM, Gottdiener JS, et al. Importance of heart failure with preserved systolic function in patients $>$ or $=65$ years of age. Am J Cardiol 2001;87:413-9.

[9] Senni M, Tribouilloy CM, Rodeheffer RJ, et al. Congestive heart failure in the community: a study of all incident cases in Olmsted County, Minnesota, in 1991. Circulation 1998;98:2282-896.

[10] Paulus WJ, Tschöpe C, Sanderson JE, et al. How to diagnose diastolic heart failure: a consensus statement on the diagnosis of heart failure with normal left ventricular ejection fraction by the Heart Failure and Echocardiography Associations of the European Society of Cardiology. Eur Heart J 2007;28(20):2539-50.

[11] Borlaug BA, Paulus WJ. Heart failure with preserved ejection fraction: athophysiology, diagnosis, and treatment. Eur Heart J. 2010; Dec 7. [Epub ahead of print] doi:10.1093/eurheartj/ehq426.

[12] Katz AM, Zile MR. New molecular mechanism in diastolic heart failure. Circulation 2006;113(16):1922-5.

[13] Zile MR, Brutsaert DL. New concepts in diastolic dysfunction and diastolic heart failure, part II: causal mechanisms and treatment. Circulation 2002;105:1503-8.

[14] Van Heerebeek L, Borbely A, Niessen HW, et al. Myocardial structure and function differ in systolic and diastolic heart failure. Circulation 2006;113:1966-73.

[15] Westermann D, Lindner D, Kasner $M$, et al. Cardiac inflammation contributes to changes in the extracellular matrix in patients with heart failure and normal ejection fraction. Circ Heart Fail 2011;4(1):44-52.

[16] Modena MG, Aveta P, Menozzi A, Rossi R. Aldosterone inhibition limits collagen synthesis and progressive left ventricular enlargement after anterior myocardial infarction. Am Heart J 2001;141(1):41-6.

[17] Paulus WJ, van Ballegoij JJ. Treatment of heart failure with normal ejection fraction: an inconvenient truth! J Am Coll Cardiol 2010 Feb 9;55(6):526-37.

[18] Yusuf S, Pfeffer MA, Swedberg K, et al. Effects of candesartan in patients with chronic heart failure and preserved left-ventricular ejection fraction: the CHARM-Preserved Trial. Lancet 2003;362(9386):777-81.

[19] Ahmed A, Rich MW, Fleg JL, et al. Effects of digoxin on morbidity and mortality in diastolic heart failure: the ancillary digitalis investigation group trial. Circulation 2006;114(5):397-403.

[20] Cleland JG, Tendera M, Adamus J, Freemantle N, Polonski LTaylor J. The perindopril in elderly people with chronic heart failure (PEP-CHF) study. Eur Heart J 2006;27(19):2338-45.

[21] Massie BM, Carson PE, McMurray JJ, et al. Irbesartan in patients with heart failure and preserved ejection fraction. N Engl J Med 2008;359(23):2456-67.

[22] Blum A, Shamburek R. The pleiotropic effects of statins on endothelial function, vascular inflammation, immunomodulation and thrombogenesis. Atherosclerosis 2009;203(2):325-30.

[23] Fukuta H, Sane DC, Brucks S, Little WC. Statin therapy may be associated with lower mortality in patients with diastolic heart failure: a preliminary report. Circulation 2005;112:357-63. 
[24] Indolfi C, Di Lorenzo E, Perrino C, et al. Hydroxymethylglutaryl coenzyme A reductase inhibitor simvastatin prevents cardiac hypertrophy induced by pressure overload and inhibits p21ras activation. Circulation 2002;106:2118-24.

[25] Loch D, Levick S, Hoey A, Brown L. Rosuvastatin attenuates hypertensioninduced cardiovascular remodeling without affecting blood pressure in DOCA-salt hypertensive rats. J Cardiovasc Pharmacol 2006;47:396.

[26] Gómez-Garre D, González-Rubio ML, Muñoz-Pacheco P, Caro-Vadillo A, Aragoncillo P, Fernández-Cruz A. Rosuvastatin added to standard heart failure therapy improves cardiac remodelling in heart failure rats with preserved ejection fraction. Eur J Heart Fail 2010;12(9):903-12.

[27] Shah R, Wang Y, Foody JM. Effects of statins, angiotensinconverting enzymeinhibitors, and $\beta$-blockers on survival in patients $\geq 65$ years of age with heart failure and preserved left ventricular systolic function. Am J Cardiol 2008;2:217-22.
[28] Chang SA, Kim YJ, Lee HW, et al. Effect of rosuvastatin on cardiac remodeling function, and progression to heart failure in hypertensive heart with established left ventricular hypertrophy. Hypertension 2009;54(3):591-7.

[29] Investigators Gissi-HF, Tavazzi L, Maggioni AP, et al. Effect of rosuvastatin in patients with chronic heart failure (the GISSI-HF trial): a randomised, doubleblind, placebo-controlled trial. Lancet 2008;372(9645):1231-9.

[30] Nishikawa H, Miura S, Zhang B, et al. Statins induce the regression of left ventricular mass in patients with angina. Circ J 2004;68:121-5.

[31] Patel R, Nagueh S, Tsybouleva N, et al. Simvastatin induces regression of cardiac hypertrophy and fibrosis and improves cardiac function in a transgenic rabbit model of human hypertrophic cardiomyopathy. Circulation 2001;104: 317-24.

[32] Zile MR. Treating diastolic heart failure with statins: "phat" chance for pleiotropic benefits. Circulation 2005;112(3):300-3. 